\title{
Defect in Synaptic Pruning of Motor Cortex Neurons is Associated with Early Perturbed Dopaminergic System
}

\author{
Bankole Oluwamolakun ${ }^{1 *}$, Ogunnusi Tolulope ${ }^{1}$, Laoye Babafemi ${ }^{1}$, Ishola Azeez ${ }^{2}$ and Poluyi Edward ${ }^{3}$ \\ ${ }^{1}$ Department of Biological Sciences, College of Sciences, Afe Babalola University, Ado-Ekiti, Ekiti State, Nigeria \\ ${ }^{2}$ Department of Anatomy, College of Medicine and Health Sciences, Afe Babalola University, Ado-Ekiti, Ekiti State, Nigeria \\ ${ }^{3}$ Department of Neurosurgery, Lagos University Teaching Hospital, Lagos State, Nigeria
}

*Corresponding author: Bankole Oluwamolakun, Department of Biological Sciences, College of Sciences, Afe Babalola University, Ado-Ekiti, Ekiti State, Nigeria, Tel: +23408084887065; E-mail: bankolem@abuad.edu.ng

Received date: August 10, 2018; Accepted date: September 14, 2018; Published date: September 21, 2018

Copyright: (c) 2018 Oluwamolakun B, et al. This is an open-access article distributed under the terms of the Creative Commons Attribution License, which permits unrestricted use, distribution and reproduction in any medium, provided the original author and source are credited.

\begin{abstract}
Background: Early stress exposure during neurodevelopmental stages has been linked to some adult neuropsychiatric disorders. The dopaminergic system which has been implicated in movement and reward system has been linked to movement and mood disorders when perturbed at early development. This study is designed to check the mechanisms involved in movement disorders such as dyskinesia, associated with early perturbed dopaminergic system in the motor cortex.
\end{abstract}

Methods: Haloperidol was used to block D2R in neonatal albino Wistar rats in utero by administering $20 \mathrm{mg} / \mathrm{kg}$ BW (intraperitoneally) to pregnant adult wistar rats $(n=8)$ in the third week of gestation. Behavioural studies such as the rotarod test were carried out on the neonatal animals $(n=5)$ to test their motor function at postnatal day twentyeight (P28). Electrophysiological recordings were carried out on the motor cortex (M1) to determine the significance of D2R inhibition on calcium neural activity. Immunofluorescence was done to demonstrate synaptic vesicle protein (SV) and microtubule associated protein kinases (MAP K) as a measure of synapses count and microtubule phosphorylation respectively.

Results: Behavioural studies showed a decline in motor function of animals exposed to haloperidol in utero compared to the control. This motor deficit was accompanied by a significant increase in the $\mathrm{Ca}^{2+}$ neural activity of the motor cortex as shown by electrophysiological recordings. Immunofluorescence staining showed there was significant increase in the number of MAPK+ and SV+ cells in the motor cortex of haloperidol exposed animals compared to the control.

Conclusion: These findings showed that early perturbation in dopaminergic system is associated with an increase in synapses and neuronal density, as well as an increase in phosphorylation of microtubules of neurons in the motor cortex.

Keywords: Dopaminergic (D2R) receptor; Motor cortex (M1); Synaptic vesicle; Microtubule; Haloperidol

List of Abbreviations: D2R: Dopaminergic receptor; M1: Motor cortex; TD: Tardive Dyskinesia; LOF: Latency of Fall; OFA: Open field Area; -D2: Exposed group; NS: Normal saline; PD: Parkinson's Disease; SV: Synaptic vesicle; MAP K: Microtubule associated protein kinase; VGAT: Vesicular GABA transporter; p: Phosphorylation

\section{Introduction}

Antipsychotics are often employed in the management of depression, schizophrenia and other neurological disorders; however, prolonged use of these drugs often results in movement disorders [1]. The primary effects of these drugs involve the inhibition of dopaminergic D2 receptor in the nigrostriatal system and cortical projections, leading to persistent involuntary movements in the face, limbs, oral region, trunk and a decline in memory function [2,3]. Dopaminergic innervations are the most prominent in the brain and generally exert their action on neuronal circuitry via a relatively slow modulation of neurotransmission [4].

Haloperidol, a typical antipsychotic medication and Dopaminergic D2 receptor blocker, has been known to induce toxicity in the motor neural system [5]. Furthermore, its major mechanism of toxic induction has been identified to involve weak binding of haloperidol to the dopamine receptive site on D2 receptor domain; this often results to receptor de-sensitivity after a prolonged duration $[6,7]$. This in effect prevents the heteromeric combination of dopaminergic D2 with D1 receptors required for calcium signaling in dopaminergic neurotransmission [8]. Furthermore, D2-D1 calcium signaling has been shown to be involved in the activation of BDNF, vesicle clearance and CamKII a activation: all of which are required for synaptic plasticity and motor neural function [2].

Previous studies have shown that haloperidol has been associated with neuronal damage and cytoskeletal rearrangement in neuroblastoma N1-E115 cells due to increased levels of oxidative stress [9]. Reactive oxygen species produce cytoskeletal collapse and an excessive phosphorylation of tau, a microtubule-associated protein that 
plays a key role in microtubule stabilization, as well as in growth cone motility and neurite formation, which are cytoskeletal phenotypes that participate in neurodevelopment [9]. Furthermore, haloperidolinduced tardive dyskinesia as well as other hyperkinetic disorders have been associated with maladaptive neural plasticity that can be expressed by functional changes such as an increase in transmitter release, receptor regulation, and synaptic plasticity or anatomical modifications such as axonal regeneration, sprouting, synaptogenesis, and neurogenesis [10]. Recent studies from human and animal models have provided evidence to the hypothesis that these phenomena likely depend on altered dopamine turnover induced by long-term drug treatment. However, it is still unclear how and where these altered mechanisms of synaptic plasticity may be localized [10].

\section{Materials}

Rabbit anti-MAP K antibody was procured from Thermo Fisher, while rabbit anti-SV antibody and other chemical reagents were procured from Abcam. Haloperidol was sourced from Kanada Pharmacy, Nigeria and re-suspended in dextrose saline. This was prepared weekly as needed and stored at $4^{\circ} \mathrm{C}$.

\section{Methods}

\section{Housing and animal handling}

Sixteen adult Wistar rats (12 females and 4 males) were purchased from the animal holding facility of Afe Babalola University Ado- Ekiti, Ekiti State, Nigeria. They were housed in standard cages with water and food given ad libitum. The animals were allowed to acclimatize to the new environment for 1 week. Animals were housed four per cage, with each cage being made up of females $n=3$ and male $n=1$. The animals were placed in such a manner for the purpose of mating and were kept under natural alternating light and darkness.

\section{Vaginal smear}

Vaginal smear was carried out on the female albino Wistar adult rats each morning to confirm mating as previously described by Marcondes et al. [11]. The smear was done by aspirating $0.04 \mathrm{~mL}$ of normal saline with rubber pipette, inserted to the vagina of the female animals. The pipette was then used to flush the vagina and aspirate back. This was then placed on a glass slide and viewed under a microscope, using 10X objective. Mating was confirmed by the presence of sperm cells in the vaginal smear. Animals that have mated where then separated into different cages for treatment.

\section{Animal treatment}

Animals in the exposed group $(\mathrm{n}=8)$ were injected (intraperitoneal) with $20 \mathrm{mg} / \mathrm{kg}$ body weight of haloperidol at $3 \mathrm{rd}$ week after fertilization.

The remaining pregnant animals $(n=4)$ were used as the control group without any treatment.

\section{Behavioural studies}

After delivery, behavioural studies were carried out on the pups' rats at postnatal day twenty-eight (P28). Five pups each were used for this protocol. Animals were taken through the Rotarod test and Open Field Test (OFT).

\section{Rotarod test}

This was done to measure motor activity as a function of latency of fall (LOF) on the treadmill. Each animal was placed on the rotating bar of the Rotarod and the rotation was measured in revolution per minute $(\mathrm{rpm})$ for test duration of 3 minutes. The duration spent on the Rotarod (before fall) was measured to determine the LOF. Subsequently, the average LOF of animals in the control and exposed group was determined and compared with each other.

\section{Open field test}

Exploratory motor function associated with prefrontal cortex activity was examined as the frequency of line crossing in an open field area (OFA). The OFT was conducted in an isolated room with a camera setup to record the activity of the animals in the OFA. In the actual test, each animal was allowed to perform the task for 3 minutes. Subsequently, the number of lines crossed by each animal for the allotted test duration was counted.

\section{Electrophysiology}

Electrophysiological recordings of extracellular calcium hyperpolarization currents were obtained from the Motor cortex (M1) using chronically implanted wire electrodes according to the method of Ishola et al. [12]. The animals were deeply anesthetized using $0.3 \mathrm{ml}$ of Ketamine (i.p.) to keep the mice immobile but awake for basal motor functions (corneal reflexes and diaphragmatic movement). Using a stereotaxic frame, the scalp was removed above the bregma to expose the cranium. Periosteal tissue was removed using hydrogen peroxide solution with cotton bud. The M1 was located using a calibrated grid to determine the position and depth (electrode length) relative to the bregma, M1 (AP: $-1.2 \mathrm{~mm} \mathrm{ML:}-2.0 \mathrm{~mm} \mathrm{DV}:+3.0 \mathrm{~mm}$ ), A small needle was used to make a hole in the cranium, following which insulated wire electrodes were inserted to the appropriate depth. The ground electrode was placed on the cranium of the contralateral side. The terminal wires of the electrodes were connected to the amplifier through small head-sockets in preparation for immobile awake recordings. The data from the amplifier (Spiker Box; Backyard Brains, Michigan, USA) was captured on the Audacity software v4.2 and analyzed in Sig View v2.1 (Signal Labs, USA) to determine the extracellular summation epoch neural activity (calcium signals) expressed as Frequency $(\mathrm{Hz})$ per unit time.

\section{Immunofluorescence}

The pups were deeply anaesthetized via ketamine overdose, and then perfused transcardially through the left ventricle using $0.9 \%$ normal saline, subsequently $4 \%$ paraformaldehyde (PFA) was introduced for perfusion fixation. The whole brain was harvested and fixed in $4 \%$ PFA overnight following which it was transferred to $4 \%$ PFA containing $30 \%$ sucrose for cryopreservation. Free floating cryostat sections $(40 \mu \mathrm{m})$ were obtained using a Leica Cryostat and stored in $10 \mathrm{mM}$ phosphate-buffered saline (PBS) at $4^{\circ} \mathrm{C}$. The sections were washed three times ( $5 \mathrm{~min}$ each) in $10 \mathrm{mM}$ PBS on a tissue rocker. Blocking was performed in normal goat serum (Vector Labs), prepared in $10 \mathrm{mM}$ PBS $+0.03 \%$ Triton-X 100, for 2 hours at room temperature. The sections were incubated in primary antibody solution overnight at 40C. Rabbit anti-MAPK (1:100 AH01202 Thermo Fisher), Rabbit anti-SV (1:100 ab11096 abcam) diluted in $10 \mathrm{mM}$ PBS, $0.03 \%$ Triton-X 100 and normal goat serum. Subsequently, the sections were washed as previously described and incubated in secondary antibody 
Citation: $\quad$ Oluwamolakun B, Tolulope O, Babafemi L, Azeez I, Edward P (2018) Defect in Synaptic Pruning of Motor Cortex Neurons is Associated with Early Perturbed Dopaminergic System. Biol Med (Aligarh) 10: 449. doi:10.4172/0974-8369.1000449

solution (Goat anti rabbit Alexa 594, Goat anti rabbit Alexa 568) diluted at 1:1000 prepared in $10 \mathrm{mM}$ PBS, $0.03 \%$ Triton-X 100 and normal goat serum at room temperature for 1 hour. Immunolabelled sections were washed and mounted on gelatin-coated slides.

\section{Confocal microscopy and cell count}

The distribution of immunolabelling in the motor cortex was imaged using Olympus Fluoview 10i confocal microscope (Olympus America, Center Valley, PA). Protein expression was determined from the confocal motor cortex images ( $n=5$ per group per protein) using Image $J$ software (NIH, USA). For each section, the count was determined per $\mu \mathrm{m}^{2}$ for $\mathrm{n}=10$ different field of views. This was done for $\mathrm{n}=5$ successive sections taken at $50 \mu \mathrm{m}$ apart.

\section{Statistical Analysis}

Data were expressed in mean \pm SEM. Statistical significance of the difference between the groups were analysed using two-way student $\mathrm{t}$ test at significant level of 0.005 .

\section{Results}

\section{Motor function declines after haloperidol exposure}

Motor function deficit was observed in animals exposed to haloperidol in utero. This was evident as the animals recorded significant decrease $(\mathrm{P}=0.0062)$ in the time spent on rotating bar (termed latency of fall LOF) compared to the unexposed animals (control) (Figure 1).

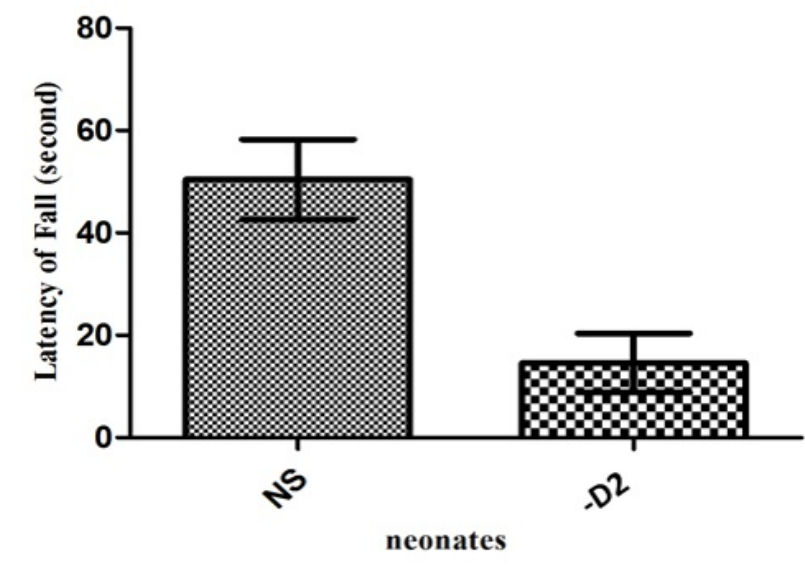

Figure 1: Graph showing a decline in the latency of fall (LOF) of animals in the exposed group (-D2) compared to the control group (NS). The latency of fall was determined on the rotarod test treadmill for a test duration of 3 minutes and animals exposed to haloperidol treatment showed a significant reduction $(\mathrm{P}=0.0062)$ in motor function compared to the animals in the control group.

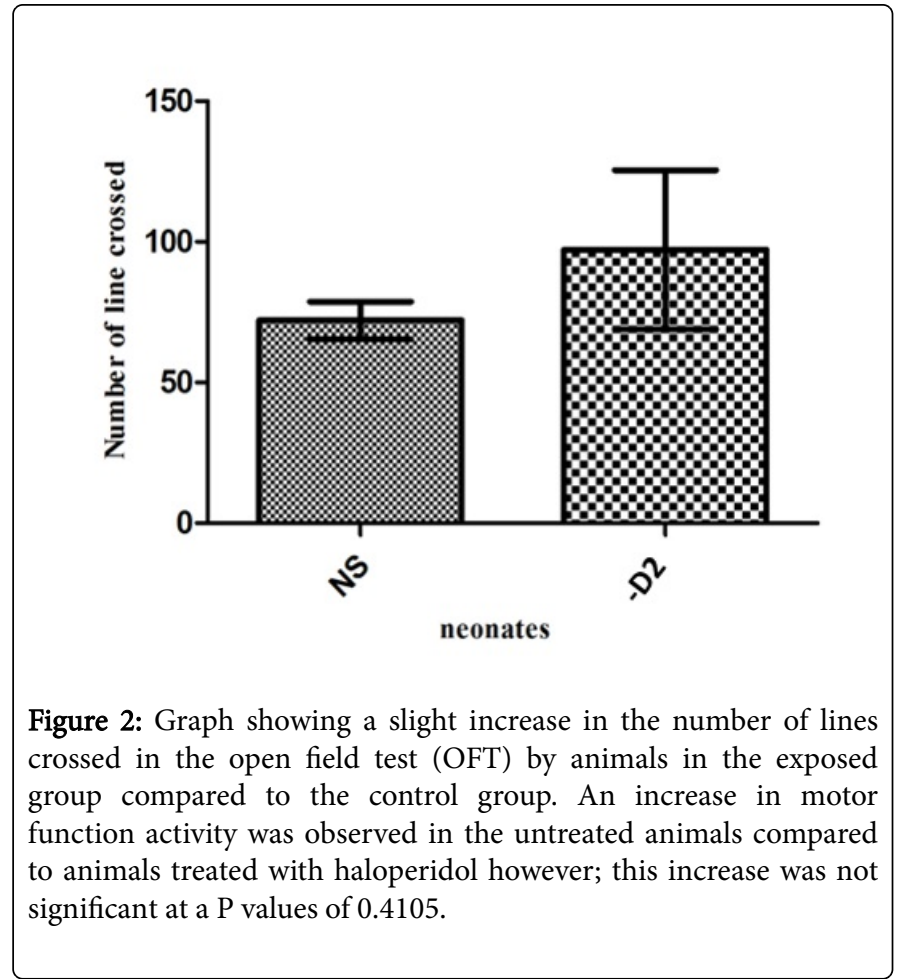

Furthermore, the open ield test (OFT) showed that the number of lines crossed which is an index of locomotor activity was increased in the haloperidol exposed animals, however, no signi icant difference was observed $(\mathrm{P}=0.4105)$ (Figure 2).

\section{Haloperidol treatment causes an increase in extracellular calcium neural activity}

Figure 3 shows a comparison between the electrophysiological recordings of extracellular hyperpolarization current obtained from the motor cortex of animals in both the control and exposed group. It was observed that there was an increase in the extracellular calcium neural activity of animals in the exposed group when compared to those in the control group, which indicates that the rate of $\mathrm{Ca}^{2+}$ movement into the neurons of animals a ter haloperidol treatment is signi icantly increased $(\mathrm{P}=0.0012)$. 
Citation: $\quad$ Oluwamolakun B, Tolulope O, Babafemi L, Azeez I, Edward P (2018) Defect in Synaptic Pruning of Motor Cortex Neurons is Associated with Early Perturbed Dopaminergic System. Biol Med (Aligarh) 10: 449. doi:10.4172/0974-8369.1000449

Page 4 of 6

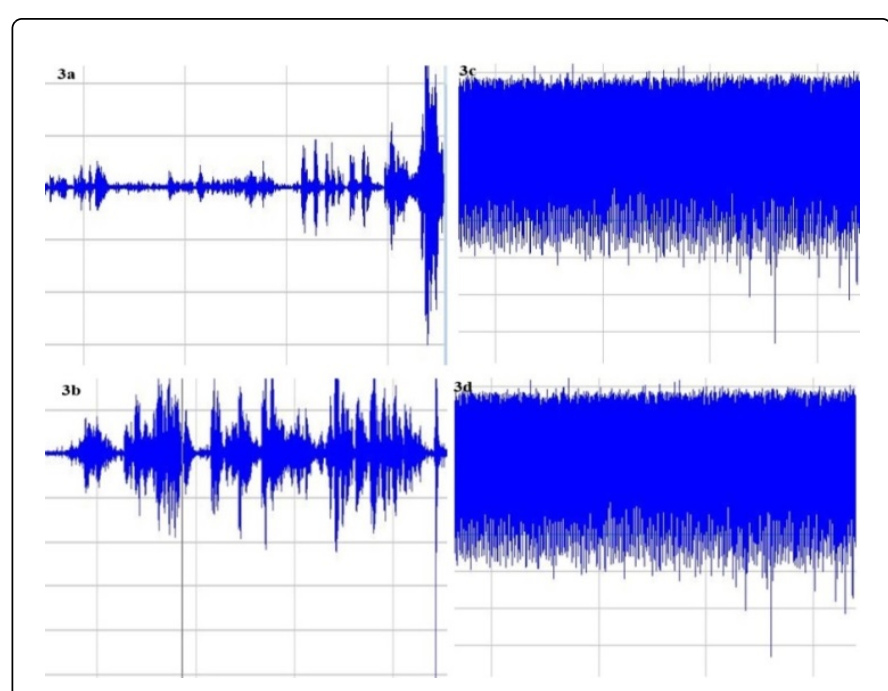

Figure 3: Electrophysiological records of M1 region of control $(a, b)$ and exposed (c, d) groups. An increase in extracellular neural activity was observed after treatment with haloperidol compared with the control group. This increase in neural activity was characterized by an increase in burst pattern after haloperidol treatment which indicates an increase in calcium signalling and the opening of calcium channels enabling the movement of $\mathrm{Ca}^{2+}$ into the neurons.

This is evident by an increase in the RMS (Hz) (Figure 4) of animals in the exposed group which is characterized by an increase in the burst pattern of neural recordings.

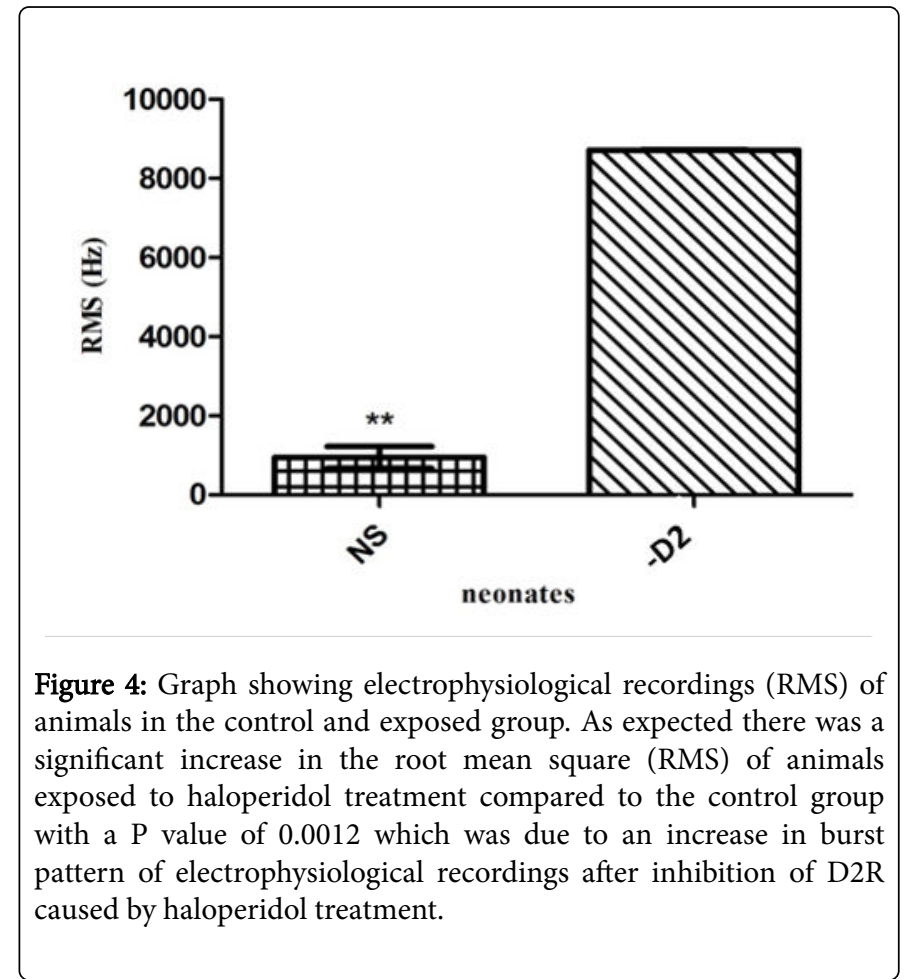

Increased synaptic activity was observed in the haloperidol exposed animals compared to control animals

Synaptic vesicle (SV) protein expression was increased in haloperidol exposed animals in utero (Figure 5a) compared to unexposed animals (Figure 5b) indicating increased synaptic activity. This expression was co-localized with VGAT (Figures $5 \mathrm{c}$ and $5 \mathrm{~d}$ ) in both the control and exposed groups, which indicated increased synaptic activity and vesicular transport in the haloperidol exposed animals compared to the control (Figures $5 \mathrm{e}$ and $5 \mathrm{f}$ ).

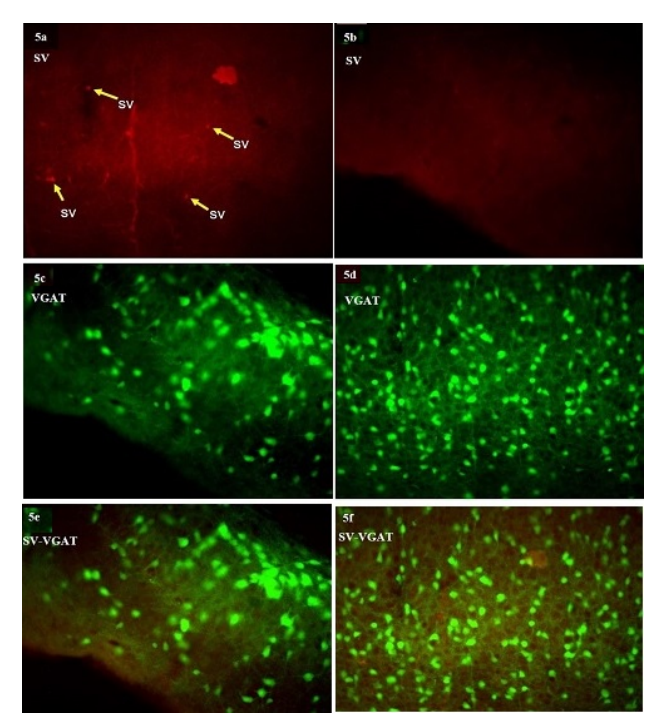

Figure 5: Immunolabeling of SV, VGAT and SV-VGAT colocalization of the M1 region of animals in the control group (a, b, c) and exposed group (d, e, f). There was an increase in synaptic vesicle (SV) protein and Vesicular GABA Transporter (VGAT) after D2R was blocked by haloperidol treatment which indicates an increase in synapses formation of motor cortex neurons and animals in the exposed group compared to the control.

Signalling molecule MAP-K expression increased in haloperidol exposed animals in utero compared with the control (Figures 6a and $6 b)$, which yielded an increase in phosphorylation of microtubules (p) when compared to the control. This expression was co-localized with VGAT (Figures $6 \mathrm{c}$ and $6 \mathrm{~d}$ ), and although MAP-K was not highly colocalized with VGAT in the control, this was not the case in haloperidol exposed animals (Figures 6e and 6f). This shows that signaling activities in the control are not necessarily related to synaptic activity unlike the haloperidol exposed animals. Furthermore, an increase was observed in the number of positively labelled neurons of animals in the exposed group when compared to the control of both SV-VGAT co-localization (Figure 7) and MAP K-VGAT colocalization (Figure 8), indicating an increase in neuronal density of animals in the exposed group. 
Citation: $\quad$ Oluwamolakun B, Tolulope O, Babafemi L, Azeez I, Edward P (2018) Defect in Synaptic Pruning of Motor Cortex Neurons is Associated with Early Perturbed Dopaminergic System. Biol Med (Aligarh) 10: 449. doi:10.4172/0974-8369.1000449

Page 5 of 6
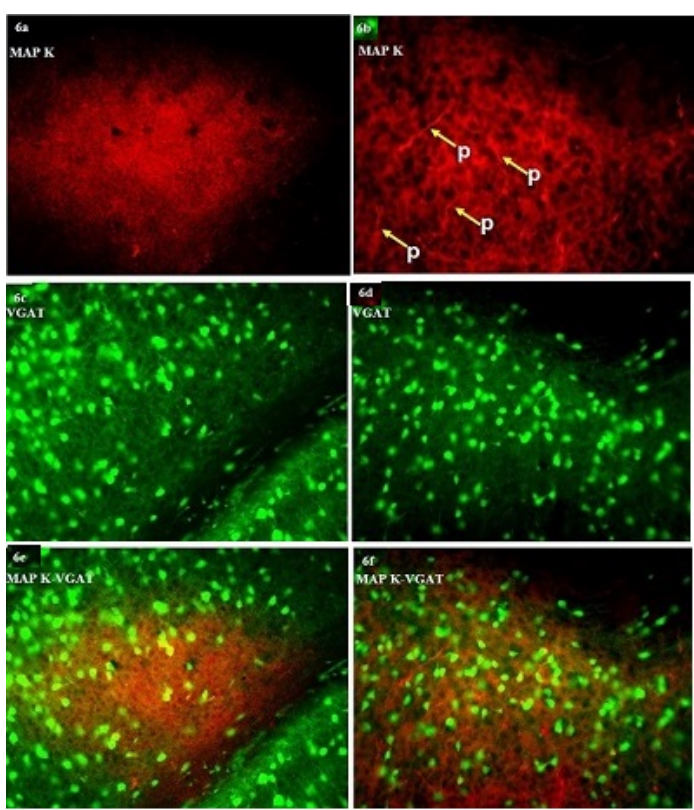

Figure 6: Immunolabeling of MAP K, VGAT and MAP K-VGAT co-localization of the M1 region of animals in the control group (a, $b, c)$ and exposed (d, e, f) group. There was increase in Microtubule Associated Protein Kinase (MAP K) and Vesicular GABA Transporter (VGAT) after animals were subjected to haloperidol treatment. This was evident by an increase in phosphorylation of microtubules of motor cortex neurons which further indicates an increase in synaptic connection of neurons in the motor cortex.

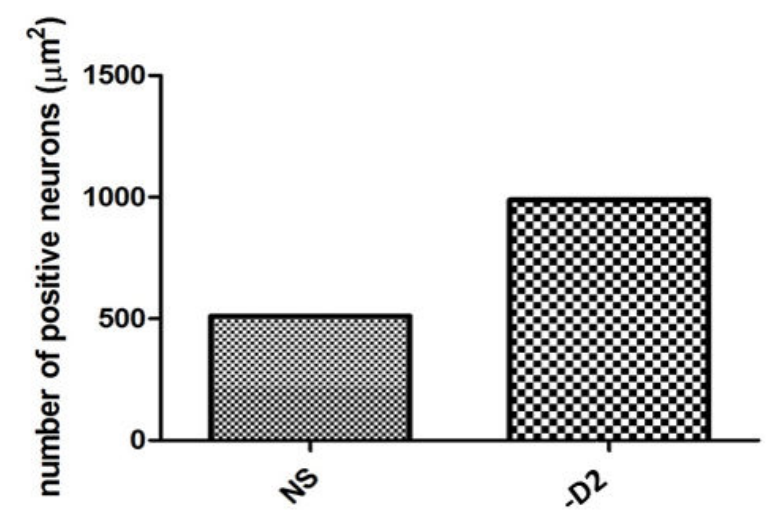

Figure 7: Graph showing positively labelled neurons of SV-VGAT co-localization of the M1 region of animals in the control and exposed group. As expected cell count showed an increase in the number of positively labelled neurons after immunofluorescence labelling with SV-VGAT in the M1 region of the motor cortex of animals exposed to haloperidol compared to the control.

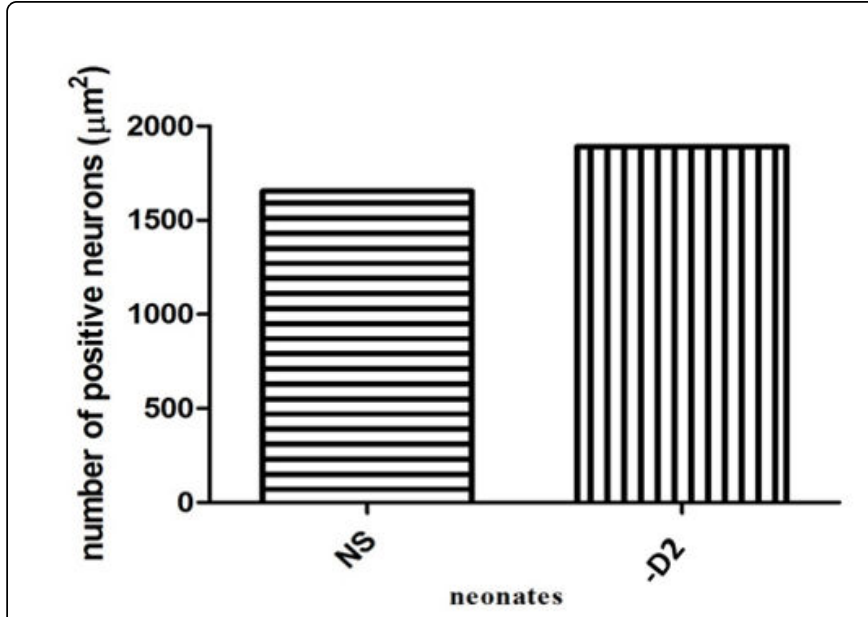

Figure 8: Graph showing positively labelled neurons of MAP KVGAT co-localization of the M1 region of animals in the control and exposed group. Cell count showed an increase in the number of positively labelled neurons after immunofluorescence labelling with MAP K-VGAT in the M1 region of the motor cortex of animals exposed to haloperidol compared to the control.

\section{Discussion}

From this study, it was observed that animals exposed to haloperidol in utero (-D2) demonstrated a slight motor function impairment, which was characterized by a decrease in the latency of fall (LOF), when compared to animals in the control group (NS). Furthermore, animals in the exposed group (-D2) exhibited an increase in motor epoch neural activity in the motor cortex which was associated with a decline in motor function. This increase in motor epoch neural activity suggests that after haloperidol treatment, there was an increase in extracellular calcium $\left(\mathrm{Ca}^{2+}\right)$ neural activity causing an inward flow of calcium into the neurons which was characterized by an increase in the root mean square (RMS), in the motor cortex when compared to the control group. Similarly, the spike train of the electrophysiological recordings showed an increase in hyperpolarization in the motor cortex of animals in the exposed group when compared to those in the control group. These findings were similar to that of Ishola et al. which records an increase in epoch neural activity of the motor cortex (M1) after haloperidol treatment characterized by an increase in the RMS and decline in motor function when it was compared to the control group (NS) [12].

Taken further, immunofluorescence labelling of VGAT and SV proteins, which are essential for synapses and neurotransmitter transport from the presynaptic membrane to the postsynaptic membrane, showed a significant increase in the number of positively labelled neurons and synapses of animals in the exposed group (-D2) when compared to the control. This was coupled with increase in synaptic vesicle protein (SV) in animals exposed to haloperidol in utero.

Microtubules also play an important role in neuron function and are partly responsible for protein and neurotransmitter transport and dynamics between neurons [13]. In order for synapses to occur, phosphorylation of the microtubule needs to take place [14]. An 
increase in the phosphorylation of microtubules in the motor cortex of animals in the exposed group was observed as opposed to the control. This increased microtubule phosphorylation suggests an increase in extracellular calcium neural activity, which corresponds with the result observed from the electrophysiological recordings. This indicates that there was increased activity of neuron and synapses formation, which may be due to neuroplasticity [15].

Furthermore, perturbation of the dopaminergic system in utero increased neuronal density coupled with synaptic formation. Studies have shown that this may be as a result of loss of neurons, whereby brain compensatory mechanism to ameliorate the effect produces more neurons and synapses [16]. During normal development, increase in synaptic formation is seen during early brain development, a process known as exuberant synaptogenesis [17]. However, synaptic pruning occurs later on in childhood and adolescents which is necessary to reshape the brain to reach adult conformation [18]. Studies have shown that children as well as adolescents with autism exhibit synaptic pruning deficit and as a result have a surplus of synapse in the brain [19].

These findings suggest that synaptic pruning may also be altered in animals exposed to haloperidol in utero as is evident by an increase in neuronal density and synapse.

\section{Conclusion}

Perturbation to dopaminergic system in utero may have a long term effect on the brain function. This may be due to a lack of, or decrease in pruning of synapse to reach adult conformation and may be the basis of dopaminergic perturbation in utero that leads to adult neuropsychiatry disorder.

\section{Acknowledgement}

The authors thank Dr Ogundele of LSU for his help on the immunofluorescence and confocal procedures, the Department of Biological Sciences and the Department of Anatomy Afe Babalola University for their support.

\section{Competing Interest}

The authors declare that they have no competing interests.

\section{Ethical Approval}

All animal handling protocols were in accordance with the Institutional Animal Care and Use Committee (IACUC) animal use guidelines and were approved by the Animal Use Ethical Committee of the Afe Babalola University, Nigeria.

\section{Authors Contributions}

$\mathrm{BO}$ and OT designed the work. BO, LB, and IA were involved in the electrophysiology and immunochemistry. BO, OT and PE contributed to the behavioural studies and helped to draft the manuscript. All authors read and approved the final manuscript.

\section{Funding}

This study was individually funded and supported by all the authors who participated and contributed to the work.

\section{References}

1. Gardos G (1999) Managing Antipsychotic-Induced Tardive Dyskinesia. Drug Saf 20: 187-193.

2. Zhang S, Xie C, Wang Q, Liu Z (2014) Interactions of CaMKII with Dopamine D2 Receptors: Roles in Levodopa-Induced Dyskinesia in 6Hydroxydopamine Lesioned Parkinson's Rats. Sci Rep 29: 6811.

3. Calabresi P, Picconi B, Tozzi A, Ghiglieri V, Di Filippo M (2014) Direct and Indirect Pathways of Basal Ganglia: A Critical Reappraisal. Nat Neurosci 17: 1022-1030.

4. Jean-Martin B, Raul RG (2011) The Physiology, Signalling, and Pharmacology of Dopamine Receptors. Pharmacol Rev 63: 182-217.

5. Haili Z, Ming Z, Manhong W, Dan X, Toshihiko N, et al. (2016) A Pharmacogenetic Discovery: Cystamine Protects Against HaloperidolInduced Toxicity and Ischemic Brain Injury. Genetics 203: 599-609.

6. Napier TC, Maslowski-Cobuzzi RJ (1994) Electrophysiological Verification of the Presence of D1 and D2 Dopamine Receptors within the Ventral Pallidum. Synapse 17: 160-166.

7. Shi WX, Smith PL, Pun CL, Millet B, Bunney BS (1997) D1-D2 Interaction in Feedback Control of Midbrain Dopamine Neurons. J Neurosci 17: 7988-7994.

8. Perreault ML, Hasbi A, O’Dowd BF, George SR (2014) Heteromeric Dopamine Receptor Signaling Complexes: Emerging Neurobiology and Disease Relevance. Neuropsychopharmacology 39: 156-168.

9. Gloria BK, Leonardo OL, Graciela JR, Gerardo RR (2010) Haloperidol causes cytoskeletal collapse in N1E-115 cells through tau hyper phosphorylation induced by oxidative stress: Implications for neurodevelopment. Euro J Pharmacol 644: 24-31.

10. Antonio C, Alfonso F, Francesca M, Giacomo K, Aldo Q (2014) Maladaptive Plasticity in Levodopa-Induced Dyskinesias and Tardive Dyskinesias: Old and New Insights on the Effects of Dopamine Receptor Pharmacology. Front Neurol 5: 49.

11. Marcondes FK, Bianchi FJ, Tanno AP (2002) Determination of the estrous cycle phases of rats: some helpful considerations. Braz J Biol 62: 609-614.

12. Ishola OA, Laoye JB, Damilola EO, Bankole OO., Mujittapha US, et al. (2015) Vitamin D3 Receptor Activation Rescued Corticostriatal Neural Activity and Improved Motor-Cognitive Function in -D2R Parkinsonian Mice Model. J Biomed Sci Eng 8: 601-615.

13. Laura P, Andrea W, Simone G, George H, Kirsten H (2017) Back to the tubule: microtubule dynamics in Parkinson's disease. Cell Mol Life Sci 74 : 409-434.

14. Travis JA, Jack AT, Stuart H (2012) Cytoskeletal Signaling: Is Memory Encoded in Microtubule Lattices by CaMKII Phosphorylation? PLoS. Comput Biol 8: e1002421.

15. Kays JL, Hurley RA, Taber KH (2012) The Dynamic Brain: Neuroplasticity and Mental Health. J Neuropsychiatry Clin Neurosci 24: 118-124.

16. Dario CA, Patrick H, Ling LC, Cheng W, Yan WY, et al. (2014) Thiol peroxidases ameliorate LRRK2 mutant-induced mitochondrial and dopaminergic neuronal degeneration in Drosophila. Hum Mol Genet 23: 3157-3165.

17. Gregory ZT, Bradley SP (2010) Normal development of Brain Circuits. Neuropsychopharmacology 35: 147-168.

18. Rosa CP, Giulia B, Francesca P, Laura M, Maria S, et al. (2011) Synaptic Pruning by Microglia Is Necessary for Normal Brain Development. Science 333: 1456-1458.

19. Columbia University Medical Center (2014) Children with autism have extra synapses in brain: May be possible to prune synapses with drug after diagnosis. ScienceDaily. 